\title{
The Hájek-Rènyi inequality and strong law of large numbers for ANA random variables
}

Mi-Hwa Ko*

\section{"Correspondence:}

songhack@wonkwang.ac.kr

Division of Mathematics and

Informational Statistics, Wonkwang

University, Jeonbuk, 570-749, Korea

\begin{abstract}
In this paper, the Hájek-Rènyi inequality and the strong law of large numbers for asymptotically negatively associated random variables are obtained. In particular, the classical Marcinkiewicz strong law of large numbers for negatively associated random variables is generalized to the case of asymptotically negative association.
\end{abstract}

MSC: $60 \mathrm{~F} 05 ; 60 \mathrm{~F} 15$

Keywords: asymptotically negative association; complete convergence; Hájek-Rènyi inequality; Marcinkiewicz strong law of large numbers

\section{Introduction}

Let $(\Omega, \mathcal{F}, \mathcal{P})$ be a probability space and $\left\{X_{n}, n \geq 1\right\}$ be a sequence of random variables defined on it.

A finite family of random variables $\left\{X_{i}, 1 \leq i \leq n\right\}$ is said to be negatively associated (NA) if for every pair of disjoint subsets $A, B \subset\{1,2, \ldots, n\}$ and any real coordinatewise nondecreasing functions $f$ on $R^{A}$ and $g$ on $R^{B}$

$$
\operatorname{Cov}\left(f\left(X_{i}, i \in A\right), g\left(X_{j}, j \in B\right)\right) \leq 0 .
$$

An infinite family of random variables is negatively associated if every finite subfamily is negatively associated. This concept was introduced by Joag-Dev and Proschan [1].

A new kind of dependence structure called asymptotically negative association was proposed by Zhang $[2,3]$ which is a useful weakening of the definition of negative association (see also Yuan and $\mathrm{Wu}[4]$ ).

Definition (Yuan and Wu [4]) A sequence $\left\{X_{n}, n \geq 1\right\}$ of random variables is said to be asymptotically negatively associated (ANA) if

$$
\rho^{-}(r)=\sup \left\{\rho^{-}(S, T): S, T \subset N, \operatorname{dist}(S, T) \geq r\right\} \rightarrow 0 \quad \text { as } r \rightarrow \infty,
$$

where

$$
\rho^{-}(S, T)=0 \vee\left\{\frac{\operatorname{Cov}\left(f\left(X_{i}, i \in S\right), g\left(X_{j}, j \in T\right)\right)}{\left(\operatorname{Var} f\left(X_{i}, i \in S\right)\right)^{\frac{1}{2}}\left(\operatorname{Var} g\left(X_{j}, j \in T\right)\right)^{\frac{1}{2}}}, f, g \in \mathcal{C}\right\},
$$

and $\mathcal{C}$ is the set of nondecreasing functions.

\section{Springer}

O2014 Ko; licensee Springer. This is an Open Access article distributed under the terms of the Creative Commons Attribution License (http://creativecommons.org/licenses/by/2.0), which permits unrestricted use, distribution, and reproduction in any medium, provided the original work is properly cited. 
It is obvious that a sequence of asymptotically negatively associated random variables is negatively associated if and only if $\rho^{-}(1)=0$. Compared to negative association, asymptotically negative association defines a strictly larger class of random variables (for detailed examples, see Zhang [2]).

Consequently, the study of the limit theorems for asymptotically negatively associated random variables is of much interest.

For example, Zhang [3] proved the central limit theorem, Wang and Lu [5] obtained some inequalities of the maximum of partial sums and weak convergence, Wang and Zhang [6] established the law of the iterated logarithm, and Yuan and $\mathrm{Wu}$ [4] showed the limiting behavior of the maximum of partial sums.

Hájek and Rènyi [7] proved that if $\left\{X_{n}, n \geq 1\right\}$ is a sequence of independent random variables with $E X_{n}=0$ and $E X_{n}^{2}<\infty, n \geq 1$, and $\left\{b_{n}, n \geq 1\right\}$ is a sequence of positive nondecreasing real numbers, then for any $\epsilon>0$ and for any positive integer $m<n$,

$$
P\left(\max _{m \leq j \leq n}\left|\frac{\sum_{i=1}^{j} X_{i}}{b_{j}}\right| \geq \epsilon\right) \leq \epsilon^{-2}\left(\sum_{j=m+1}^{n} \frac{E X_{j}^{2}}{b_{j}^{2}}+\sum_{j=1}^{m} \frac{E X_{j}^{2}}{b_{m}^{2}}\right) .
$$

Since then, this inequality has been of concern for more and more authors (e.g., Chow [8] and Gan [9] for martingales, Liu et al. [10] for negatively associated random variables and Kim et al. [11] for asymptotically almost negatively associated random variables).

Inspired by Kim et al. [11], we will obtain the Hájek-Rènyi inequality type for asymptotically negatively associated random variables and prove the strong law of large numbers by using this inequality.

\section{Hájek-Rènyi inequality for ANA random variables}

Lemma 2.1 (Yuan and Wu [4]) Let $\left\{X_{n}, n \geq 1\right\}$ be a sequence of asymptotically negatively associated $(A N A)$ random variables and $\left\{a_{n}, n \geq 1\right\}$ a sequence of positive numbers. Then $\left\{a_{n} X_{n}, n \geq 1\right\}$ is still a sequence of ANA random variables.

From Wang and Lu's [5] Rosenthal type inequality for asymptotically negatively associated random variables we obtain the following.

Lemma 2.2 Let $0 \leq r<\frac{1}{12}$ and $N$ be a positive integer. Let $\left\{X_{n}, n \geq 1\right\}$ be a sequence of asymptotically negatively associated random variables with $\rho^{-}(N) \leq r, E X_{n}=0$, and $E X_{n}^{2}<\infty$. Then, for all $n \geq 1$ there is a positive constant $D=D(2, N, r)$ such that

$$
E \max _{1 \leq i \leq n}\left|\sum_{j=1}^{i} X_{j}\right|^{2} \leq D\left(\sum_{j=1}^{n} E X_{j}^{2}\right)
$$

Theorem 2.3 Let $\left\{b_{n}, n \geq 1\right\}$ be a sequence of positive nondecreasing real numbers and $\left\{X_{1}, \ldots, X_{n}\right\}$ a sequence of mean zero, square integrable ANA random variables. Let $\sigma_{k}^{2}=$ $E X_{k}^{2}$. Then, for $\epsilon>0$

$$
P\left\{\max _{1 \leq k \leq n}\left(\frac{\sum_{i=1}^{k} X_{i}}{b_{k}}\right)>\epsilon\right\} \leq 4 D \epsilon^{-2} \sum_{k=1}^{n} \frac{\sigma_{k}^{2}}{b_{k}^{2}}
$$

where $D$ is a positive constant defined in Lemma 2.2 . 
Proof First note that $\left\{\frac{X_{1}}{b_{1}}, \ldots, \frac{X_{n}}{b_{n}}\right\}$ is a sequence of mean zero, square integrable ANA random variables by Lemma 2.1. Thus $\left\{\frac{X_{1}}{b_{1}}, \ldots, \frac{X_{n}}{b_{n}}\right\}$ satisfies (1.2) for all coordinatewise increasing continuous functions $f$ and $g$. Without loss of generality, set $b_{0}=0$. Since

$$
b_{k}^{-1} \sum_{j=1}^{k}\left(b_{j}-b_{j-1}\right)=1
$$

we get

$$
\sum_{j=1}^{k} X_{j}=\sum_{j=1}^{k}\left(\frac{X_{j}}{b_{j}} \sum_{i=1}^{j}\left(b_{i}-b_{i-1}\right)\right)=\sum_{i=1}^{k}\left(b_{i}-b_{i-1}\right) \sum_{i \leq j \leq k} \frac{X_{j}}{b_{j}}
$$

and

$$
\left\{\left|\frac{\sum_{j=1}^{k} X_{j}}{b_{k}}\right| \geq \epsilon\right\} \subset\left\{\max _{1 \leq i \leq k}\left|\sum_{i \leq j \leq k} \frac{X_{j}}{b_{j}}\right| \geq \epsilon\right\}
$$

From (2.3) we have

$$
\begin{aligned}
\left\{\max _{1 \leq k \leq n}\left|\frac{\sum_{j=1}^{k} X_{j}}{b_{k}}\right| \geq \epsilon\right\} & \subset\left\{\max _{1 \leq k \leq n} \max _{1 \leq i \leq k}\left|\sum_{i \leq j \leq k} \frac{X_{j}}{b_{j}}\right| \geq \epsilon\right\}=\left\{\max _{1 \leq i \leq k \leq n}\left|\sum_{j \leq k} \frac{X_{j}}{b_{j}}-\sum_{j<i} \frac{X_{j}}{b_{j}}\right| \geq \epsilon\right\} \\
& \subset\left\{\max _{1 \leq k \leq n}\left|\sum_{1 \leq j \leq k} \frac{X_{j}}{b_{j}}\right| \geq \frac{\epsilon}{2}\right\} .
\end{aligned}
$$

Hence by Lemma 2.2 the desired result (2.2) follows.

From Theorem 2.3, we can get the following more generalized Hájek-Rènyi type inequality.

Theorem 2.4 Let $\left\{b_{n}, n \geq 1\right\}$ be a sequence of positive nondecreasing real numbers. Let $0 \leq r<\frac{1}{12}$ and $N$ be a positive integer. Let $\left\{X_{n}, n \geq 1\right\}$ be a sequence of mean zero and square integrable ANA random variables with $\rho^{-}(N) \leq r$ and $E X_{n}^{2}<\infty$. Let $\sigma_{k}^{2}=E X_{k}^{2}$, $k \geq 1$. Then, for $\epsilon>0$ and for any positive integer $m<n$ we have

$$
P\left(\max _{m \leq k \leq n}\left|\frac{\sum_{j=1}^{k} X_{j}}{b_{k}}\right| \geq \epsilon\right) \leq 16 D \epsilon^{-2}\left(\sum_{j=m+1}^{n} \frac{\sigma_{j}^{2}}{b_{j}^{2}}+\sum_{j=1}^{m} \frac{\sigma_{j}^{2}}{b_{m}^{2}}\right),
$$

where D is a positive constant defined in Lemma 2.2.

Proof By Theorem 2.3 we have

$$
\begin{aligned}
& P\left\{\max _{m \leq k \leq n}\left|\frac{\sum_{j=1}^{k} X_{j}}{b_{k}}\right| \geq \epsilon\right\} \\
& \quad \leq P\left\{\left|\frac{\sum_{j=1}^{m} X_{j}}{b_{m}}\right| \geq \frac{\epsilon}{2}\right\}+P\left\{\max _{m+1 \leq k \leq n}\left|\frac{\sum_{j=m+1}^{k} X_{j}}{b_{k}}\right| \geq \frac{\epsilon}{2}\right\}
\end{aligned}
$$




$$
\begin{aligned}
& \leq P\left\{\frac{1}{b_{m}} \max _{1 \leq k \leq m}\left|\sum_{j=1}^{k} X_{j}\right| \geq \frac{\epsilon}{2}\right\}+P\left\{\max _{m+1 \leq k \leq n}\left|\frac{\sum_{j=m+1}^{k} X_{j}}{b_{k}}\right| \geq \frac{\epsilon}{2}\right\} \\
& \leq 16 D \epsilon^{-2}\left(\sum_{j=m+1}^{n} \frac{\sigma_{j}^{2}}{b_{j}^{2}}+\sum_{j=1}^{m} \frac{\sigma_{j}^{2}}{b_{m}^{2}}\right) .
\end{aligned}
$$

Hence the proof is complete.

\section{Strong law of large numbers for ANA random variables}

Using the Hájek-Rènyi inequality for ANA random variables we will prove the strong law of large number for ANA random variables.

Theorem 3.1 Let $0 \leq r<\frac{1}{12}$ and $N$ be a positive integer. Let $\left\{b_{n}, n \geq 1\right\}$ be a sequence of positive nondecreasing real numbers and $\left\{X_{n}, n \geq 1\right\}$ a sequence of mean zero, square integrable random variables with $\rho^{-}(N) \leq r$ and $E X_{n}^{2}<\infty$. Let $\sigma_{k}^{2}=E X_{k}^{2}, k \geq 1$, and $S_{n}=$ $\sum_{i=1}^{n} X_{i}$. Assume

$$
\sum_{k=1}^{\infty} \frac{\sigma_{k}^{2}}{b_{k}^{2}}<\infty
$$

Then, for any $0<p<2$

(A) $E \sup _{n}\left(\left|S_{n}\right| / b_{n}\right)^{p}<\infty$,

(B) $0<b_{n} \uparrow \infty$ implies $S_{n} / b_{n} \rightarrow 0$ a.s. as $n \rightarrow \infty$.

Proof (A) Note that

$$
E\left(\sup _{n} \frac{\left|S_{n}\right|}{b_{n}}\right)^{p}<\infty \Leftrightarrow \int_{1}^{\infty} P\left(\sup _{n} \frac{\left|S_{n}\right|}{b_{n}}>t^{\frac{1}{p}}\right) d t<\infty .
$$

By Theorem 2.3, it follows from (3.1) that

$$
\begin{aligned}
\int_{1}^{\infty} P\left(\sup _{n} \frac{\left|S_{n}\right|}{b_{n}}>t^{\frac{1}{p}}\right) d t & \leq 4 D \lim _{n \rightarrow \infty} \int_{1}^{\infty} t^{-2 / p} d t \sum_{k=1}^{n} \frac{\sigma_{k}^{2}}{b_{k}^{2}} \\
& =4 D \lim _{n \rightarrow \infty} \sum_{k=1}^{n} \frac{\sigma_{k}^{2}}{b_{k}^{2}} \int_{1}^{\infty} t^{-\frac{2}{p}} d t<\infty
\end{aligned}
$$

where $D$ is a positive constant defined in Lemma 2.2.

Hence the proof of $(\mathrm{A})$ is complete.

(B) By Theorem 2.4 we get

$$
P\left(\max _{1 \leq k \leq n} \frac{\left|S_{k}\right|}{b_{k}} \geq \epsilon\right) \leq 16 D \epsilon^{-2}\left(\sum_{j=m+1}^{n} \frac{\sigma_{j}^{2}}{b_{j}^{2}}+\sum_{j=1}^{m} \frac{\sigma_{j}^{2}}{b_{m}^{2}}\right) .
$$

But by assumption (3.1) we have

$$
P\left\{\sup _{k \geq m} \frac{\left|S_{k}\right|}{b_{k}} \geq \epsilon\right\}=\lim _{n \rightarrow \infty} P\left\{\max _{m \leq k \leq n} \frac{\left|S_{k}\right|}{b_{k}} \geq \epsilon\right\} \leq 16 D \epsilon^{-2}\left(\sum_{j=m+1}^{\infty} \frac{\sigma_{j}^{2}}{b_{j}^{2}}+\sum_{j=1}^{m} \frac{\sigma_{j}^{2}}{b_{m}^{2}}\right) .
$$


By the Kronecker lemma and (3.1) we get

$$
\sum_{j=1}^{m} \frac{\sigma_{j}^{2}}{b_{m}^{2}} \rightarrow 0 \quad \text { as } m \rightarrow \infty .
$$

Hence, by combining (3.1), (3.2), and (3.3) we have

$$
\lim _{n \rightarrow \infty} P\left\{\sup _{k \geq n} \frac{\left|S_{k}\right|}{b_{k}} \geq \epsilon\right\}=0
$$

i.e., $S_{n} / b_{n} \rightarrow 0$ a.s. as $n \rightarrow \infty$.

Corollary 3.2 Let $0 \leq r<\frac{1}{12}$ and $N$ be a positive integer. Let $\left\{X_{n}, n \geq 1\right\}$ be a sequence of mean zero, square integrable ANA random variables with $\rho^{-}(N) \leq r$ and $E X_{n}^{2}<\infty$. Then, for $0<t<2$

$$
P\left(\sup _{k \geq m} \frac{\left|S_{k}\right|}{k^{1 / t}} \geq \epsilon\right) \leq 4 D \epsilon^{-2} \frac{2}{2-t} \sup _{k} \sigma_{k}^{2} m^{(t-2) / t}
$$

for all $\epsilon \geq 0$ and $m \geq 1$, where $D$ is a constant defined in Lemma 2.2,

$$
S_{n}=\sum_{j=1}^{n} X_{j} \quad \text { and } \quad \sigma_{n}^{2}=E X_{n}^{2}, \quad n \geq 1
$$

Corollary 3.3 Let $0 \leq r<\frac{1}{12}$ and $N$ be a positive integer. Let $\left\{X_{n}, n \geq 1\right\}$ be a sequence of mean zero and square integrable ANA random variables with $\rho^{-}(N) \leq r$ and $E X_{n}^{2}<\infty$. Assume that

$$
\sup _{n} \sigma_{n}^{2}<\infty
$$

where $\sigma_{n}^{2}=E X_{n}^{2}, n \geq 1$. Then, for $0<t<2$

(A) $\left(S_{n} / n^{1 / t}\right) \rightarrow 0$ a.s. as $n \rightarrow \infty$,

(B) $E \sup _{n}\left(\left|S_{n}\right| / n^{1 / t}\right)^{p}<\infty$ for any $0<p<2$, where $S_{n}=\sum_{j=1}^{n} X_{j}$.

Finally, we consider almost convergence for weighted sums of ANA random variables as applications of Theorem 3.1.

Theorem 3.4 Let $0 \leq r<\frac{1}{12}$ and $N$ be a positive integer. Let $\left\{a_{n i}, 1 \leq i \leq n, n \geq 1\right\}$ be an array of real numbers with $a_{n i}=0, i>n, \sup _{n \geq 1} \sum_{i=1}^{n}\left|a_{n i}\right|<\infty$, and $\left\{b_{n}, n \geq 1\right\}$ be a sequence of positive nondecreasing real numbers such that $0<b_{n} \uparrow \infty$ and let $\left\{X_{n}, n \geq 1\right\}$ be a sequence of mean zero, square integrable ANA random variables satisfying $\rho^{-}(N) \leq r$, $E X_{n}^{2}<\infty$, and (3.1). Then

$$
\sum_{i=1}^{n} \frac{a_{n i} X_{i}}{b_{n}} \rightarrow 0 \quad \text { a.s. as } n \rightarrow \infty .
$$


Proof Define

$$
T_{n}=\sum_{i=1}^{n} \frac{X_{i}}{b_{n}}, \quad c_{n i}=\frac{b_{i}}{b_{n}}\left(a_{n i}-a_{n i+1}\right) \quad \text { for } 1 \leq i \leq n .
$$

Then we obtain

$$
\begin{aligned}
& c_{n n}=a_{n n}, \\
& T_{n} \rightarrow 0 \quad \text { as } n \rightarrow \infty \text { by Theorem 3.1(B), } \\
& \sum_{i=1}^{n} \frac{a_{n i} X_{i}}{b_{n}}=\sum_{i=1}^{n} c_{n i} T_{i}, \\
& \sum_{i=1}^{n}\left|c_{n i}\right| \leq 2 \sup _{n \geq 1} \sum_{i=1}^{n}\left|a_{n i}\right|<\infty,
\end{aligned}
$$

and

$$
\lim _{n \rightarrow \infty}\left|c_{n i}\right|=0 \quad \text { for every fixed } i
$$

Note that if an array of real numbers $\left\{c_{n i}, 1 \leq i \leq n, n \geq 1\right\}$ satisfies $\sum_{i=1}^{n}\left|c_{n i}\right|<\infty$ and $\lim _{n \rightarrow \infty}\left|c_{n i}\right|=0$ for every fixed $i$ then, for every sequence of real numbers $d_{n}$ with $d_{n} \rightarrow 0$ as $n \rightarrow \infty$

$$
\sum_{i=1}^{n} c_{n i} d_{i} \rightarrow 0 \quad \text { as } n \rightarrow \infty
$$

(See Kim et al. [11] for more details.)

Hence, from the above fact and (3.5)-(3.9), the desired result (3.4) follows.

Theorem 3.5 Let $0 \leq r<\frac{1}{12}$ and $N$ be a positive integer. Let $\left\{a_{n i}, 1 \leq i \leq n, n \geq 1\right\}$ be an array of real numbers with $a_{n i}=0, i>n, \sup _{n \geq 1} \sum_{i=1}^{n}\left|a_{n i}\right|<\infty$, and $\left\{b_{n}, n \geq 1\right\}$ be a sequence of positive nondecreasing real numbers such that $0<b_{n} \uparrow \infty$ and let $\left\{X_{n}, n \geq 1\right\}$ be a sequence of mean zero, square integrable ANA random variables with $\rho^{-}(N) \leq r$ and $\sup _{n} \sigma_{n}^{2}<\infty$, where $\sigma_{n}^{2}=E X_{n}^{2}, n \geq 1$. Then, for some $0<t<2$

$$
\sum_{i=1}^{n} \frac{a_{n i} X_{i}}{n^{1 / t}} \rightarrow 0 \quad \text { a.s. as } n \rightarrow \infty
$$

Proof By putting $b_{n}=n^{1 / t}$ from Corollary 3.3 and Theorem 3.4, the result follows and the proof is omitted.

Now we prove the Marcinkiewicz strong law of large numbers for ANA random variables by using Theorem 3.1. The method of proof is the same as that used in the classical Marcinkiewicz strong law of large numbers for i.i.d. random variables (see Stout [12, Theorem 3.2.3]). 
Theorem 3.6 Let $0 \leq r<\frac{1}{12}$ and $N$ be a positive integer. Let $\left\{X_{n}, n \geq 1\right\}$ be a sequence of identically distributed ANA random variables with $E X_{1}=0, E\left|X_{1}\right|^{t}<\infty$ for some $0<t<2$ and $\rho^{-}(N) \leq r$. Then

$$
\sum_{j=1}^{n} \frac{X_{j}}{n^{1 / t}} \rightarrow 0 \quad \text { a.s. as } n \rightarrow \infty
$$

Sketch of proof To prove (3.10) it suffices to show that

$$
\sum_{j=1}^{n} \frac{X_{j}^{+}}{n^{\frac{1}{t}}} \rightarrow 0 \quad \text { a.s. as } n \rightarrow \infty
$$

and

$$
\sum_{j=1}^{n} \frac{X_{j}^{-}}{n^{\frac{1}{t}}} \rightarrow 0 \quad \text { a.s. as } n \rightarrow \infty,
$$

where $X_{j}^{+}=\max \left(X_{j}, 0\right)$ and $X_{j}^{-}=\max \left(-X_{j}, 0\right)$.

Note that $\left\{X_{j}^{+}, j \geq 1\right\}$ and $\left\{X_{j}^{-}, j \geq 1\right\}$ are sequences of identically distributed ANA random variables. We only show (3.11). Equation (3.12) can be proved similarly.

Set $Y_{j}=X_{j}^{+} \wedge n^{\frac{1}{t}}, j=1,2, \ldots, n$. Then $\left\{Y_{j}, 1 \leq j \leq n\right\}$ is a sequence of identically distributed ANA random variables.

Note that $E\left|X_{1}\right|^{t}<\infty \Leftrightarrow \sum_{n=1}^{\infty} P\left(\left|X_{1}\right|>n^{\frac{1}{t}}\right)<\infty$.

$$
P\left(Y_{j} \neq X_{j}^{+}\right)=P\left(X_{1}^{+} \wedge n^{\frac{1}{t}} \neq X_{1}^{+}\right) \leq P\left(X_{1}^{+}>n^{\frac{1}{t}}\right) \leq P\left(\left|X_{1}\right|>n^{\frac{1}{t}}\right) .
$$

So

$$
P\left(Y_{j} \neq X_{j}^{+} \text {i.o. }\right)=0 \text {. }
$$

We will prove

$$
n^{-\frac{1}{t}} \sum_{j=1}^{n} E Y_{j} \rightarrow 0 \quad \text { a.s. as } n \rightarrow \infty
$$

Notice that

$$
\begin{aligned}
\sum_{n=1}^{\infty} \frac{E Y_{n}}{n^{\frac{1}{t}}} & =\sum_{n=1}^{\infty} n^{-\frac{1}{t}}\left(E X_{1}^{+} I\left(X_{1}^{+} \leq n^{\frac{1}{t}}\right)+n^{\frac{1}{t}} P\left(X_{1}^{+}>n^{\frac{1}{t}}\right)\right) \\
& =\sum_{n=1}^{\infty} n^{-\frac{1}{t}} E X_{1}^{+} I\left(X_{1}^{+} \leq n^{\frac{1}{t}}\right)+\sum_{n=1}^{\infty} P\left(X_{1}^{+}>n^{\frac{1}{t}}\right) \\
& \leq \sum_{n=1}^{\infty} n^{-\frac{1}{t}} \sum_{k=1}^{n} E X_{1}^{+} I\left((k-1)^{\frac{1}{t}}<X_{1}^{+} \leq k^{\frac{1}{t}}\right)+\sum_{n=1}^{\infty} P\left(\left|X_{1}\right|>n^{\frac{1}{t}}\right) \\
& \leq \sum_{k=1}^{\infty} E X_{1}^{+} I\left((k-1)^{\frac{1}{t}}<X_{1}^{+} \leq k^{\frac{1}{t}}\right) \sum_{n=k}^{\infty} n^{-\frac{1}{t}}+E\left|X_{1}\right|^{t}
\end{aligned}
$$




$$
\begin{aligned}
& \leq C \sum_{k=1}^{\infty} k^{-\frac{1}{t}+1} E X_{1}^{+} I\left((k-1)^{\frac{1}{t}}<X_{1}^{+} \leq k^{\frac{1}{t}}\right)+E\left|X_{1}\right|^{t} \\
& \leq C E\left|X_{1}\right|^{t}<\infty .
\end{aligned}
$$

By Kronecker's lemma and (3.15) we see that (3.14) is true.

We also have

$$
\begin{aligned}
& \sum_{n=1}^{\infty} n^{-\frac{2}{t}} E\left(Y_{n}-E Y_{n}\right)^{2} \\
& \leq C \sum_{n=1}^{\infty} n^{-\frac{2}{t}} E Y_{n}^{2} \\
& =C \sum_{n=1}^{\infty} n^{-\frac{2}{t}} E\left(X_{1}^{+} \wedge n^{\frac{1}{t}}\right)^{2} \\
& \leq C \sum_{n=1}^{\infty} n^{-\frac{2}{t}} E\left(X_{1}^{+}\right)^{2} I\left(X_{1}^{+} \leq n^{\frac{1}{t}}\right)+\sum_{n=1}^{\infty} P\left(X_{1}^{+}>n^{\frac{1}{t}}\right) \\
& \quad \leq C \sum_{n=1}^{\infty} n^{-\frac{2}{t}} \sum_{k=1}^{n} E\left(X_{1}^{+}\right)^{2} I\left((k-1)^{\frac{1}{t}}<X_{1}^{+} \leq k^{\frac{1}{t}}\right)+E\left|X_{1}\right|^{t} \\
& \quad=C \sum_{k=1}^{\infty} E\left(X_{1}^{+}\right)^{2} I\left((k-1)^{\frac{1}{t}}<X_{1}^{+} \leq k^{\frac{1}{t}}\right) \sum_{n=k}^{\infty} n^{-\frac{2}{t}}+E\left|X_{1}\right|^{t} \\
& \quad \leq C \sum_{k=1}^{\infty} k^{-\frac{2}{t}+1} E\left(X_{1}^{+}\right)^{2} I\left((k-1)^{\frac{1}{t}}<X_{1}^{+} \leq k^{\frac{1}{t}}\right)+E\left|X_{1}\right|^{t} \\
& \quad \leq C \sum_{k=1}^{\infty} k^{-\frac{2}{t}+1} k^{\frac{2}{t}-1} E\left(X_{1}^{+}\right)^{t} I\left((k-1)^{\frac{1}{t}}<X_{1}^{+} \leq k^{\frac{1}{t}}\right)+E\left|X_{1}\right|^{t} \\
& \leq C E\left|X_{1}\right|^{t}<\infty .
\end{aligned}
$$

By Theorem 3.1 and (3.13)-(3.16) the proof of (3.11) is complete.

Theorem 3.7 Let $0 \leq r<\frac{1}{12}$ and $N$ be a positive integer. Let $\left\{a_{n i}, 1 \leq i \leq n, n \geq 1\right\}$ be an array of real numbers with $\sup _{n \geq 1} \sum_{i=1}^{n}\left|a_{n i}\right|<\infty$ and let $\left\{X_{n}, n \geq 1\right\}$ be a sequence of identically distributed ANA random variables with random variables with $\rho^{-}(N) \leq r, E X_{1}=0$, and $E\left|X_{1}\right|^{t}<\infty$ for $0<t<2$. Then, for some $0<t<2$

$$
\sum_{i=1}^{n} \frac{a_{n i} X_{i}}{n^{1 / t}} \rightarrow 0 \quad \text { a.s. as } n \rightarrow \infty
$$

Proof Basically, using the ideas in the proof of Theorem 3.4 and Theorem 3.6, we can obtain (3.17) and the proof is omitted. 


\section{Acknowledgements}

The author wishes to thank the editor and the referees for their valuable comments. This paper was supported by Wonkwang University in 2014.

Received: 27 February 2014 Accepted: 5 December 2014 Published: 18 Dec 2014

\section{References}

1. Joag-Dev, K, Proschan, F: Negative association of variables with applications. Ann. Stat. 11, 286-295 (1983)

2. Zhang, LX: A functional central limit theorem for asymptotically negatively dependent random fields. Acta Math. Hung. 86, 237-259 (2000)

3. Zhang, LX: Central limit theorems for asymptotically negative dependent random field. Acta Math. Sin. Engl. Ser. 16 $691-710(2000)$

4. Yuan, DM, Wu, XS: Limiting behavior of the maximum of the partial sum for asymptotically negatively associated random variables under residual Cesàro alpha-integrability assumption. J. Stat. Plan. Inference 140, 2395-2402 (2010)

5. Wang, JF, Lu, FB: Inequalities of maximum partial sums and weak convergence for a class of weak dependent random variables. Acta Math. Sin. Engl. Ser. 23, 127-136 (2006)

6. Wang, JF, Zhang, LX: A Berry-Esseen theorem and a law of the iterated logarithm for asymptotically negatively associated sequences. Acta Math. Sin. Engl. Ser. 22, 693-700 (2007)

7. Hájek, J, Rènyi, A: Generalization of an inequality of Kolmogorov. Acta Math. Acad. Sci. Hung. 6, 281-283 (1955)

8. Chow, YS: A martingale inequality and the law of large numbers. Proc. Am. Math. Soc. 11, 107-111 (1960)

9. Gan, S: The Hájek-Rènyi inequality for Banach space valued martingales and the $p$ smoothness of Banach spaces. Stat. Probab. Lett. 32, 245-248 (1997)

10. Liu, J, Gan, S, Chen, P: The Hájek-Rènyi inequality for the NA random variables and its application. Stat. Probab. Lett. 49, 99-105 (1999)

11. Kim, TS, Ko, MH, Lee, IH: On the strong laws for asymptotically almost negatively associated random variables. Rocky Mt. J. Math. 34, 979-988 (2004)

12. Stout, WF: Almost Sure Convergence. Academic Press, New York (1972)

10.1186/1029-242X-2014-521

Cite this article as: Ko: The Hájek-Rènyi inequality and strong law of large numbers for ANA random variables. Journal of Inequalities and Applications 2014, 2014:521

\section{Submit your manuscript to a SpringerOpen ${ }^{\circ}$ journal and benefit from:}

- Convenient online submission

- Rigorous peer review

- Immediate publication on acceptance

- Open access: articles freely available online

- High visibility within the field

- Retaining the copyright to your article 